\title{
Bandwidth Behavior of Closely Spaced Aperture-Coupled Reflectarrays
}

\author{
F. Venneri, S. Costanzo, and G. Di Massa \\ Dipartimento di Elettronica, Informatica e Sistemistica, Università della Calabria, 87036 Rende, Italy \\ Correspondence should be addressed to S. Costanzo, costanzo@deis.unical.it
}

Received 2 March 2012; Revised 7 June 2012; Accepted 14 June 2012

Academic Editor: Raphaël Gillard

Copyright () 2012 F. Venneri et al. This is an open access article distributed under the Creative Commons Attribution License, which permits unrestricted use, distribution, and reproduction in any medium, provided the original work is properly cited.

The bandwidth features of reflectarray antennas are analyzed by examining in detail the phase errors due to the compensation mechanism for spatial phase delays. A bandwidth estimation rule is defined, taking into account the combined effects due to the overall antenna geometry and the frequency response of the single reflectarray element. An aperture-coupled reflectarray configuration with reduced interelement spacing is considered as broadband solution for the implementation of small reflectarrays. A $20 \mathrm{GHz}$ aperture-coupled element is synthesized for the design of a $12 \lambda$ diameter reflectarray, showing a simulated $1 \mathrm{~dB}$ gain bandwidth of $23 \%$.

\section{Introduction}

Reflectarray antennas offer many advantages over traditional reflector antennas. They combine the best features of microstrip technology with those related to parabolic reflectors. As a matter of fact, reflectarrays are low profile antennas, characterized by a cheaper fabrication process, also offering improved efficiencies due to the spatial feeding approach. As counterpart to the above advantages, reflectarrays are usually constrained to operate over a small frequency band, mainly caused by the limited bandwidth of microstrip radiators and the frequency dependence of the spatial phase delay in the paths from feed to array elements. This last effect is dominant for reflectarrays with large aperture diameters $D$ and small focal distances $F$ from the feed, resulting in reduced $F / D$ ratios (typically $F / D \leq 0.6$ ). Conversely, in the case of reflectarrays with moderate aperture dimension and $F / D$ ratio greater than 0.6 , the dominant factor limiting the antenna bandwidth is the frequency band of the single radiator.

Different solutions have been proposed to improve the bandwidth performances of reflectarray antennas.

A first class of solutions, essentially based on the exact compensation of the phase error accumulated in the different feed-element paths, comprises the use of real delay lines
[1] and stacked configuration based on the use of a threelayer reflectarray with different size patches [2]. A piecewise planar reflectarray configuration is also proposed in [3], which approximates the parabolic surface thus introducing a geometric compensation of the feed-elements path delay, just as in the case of parabolic reflectors.

A second group of solutions focuses on the design of broadband reflectarray elements. A complete overview of all existing wideband reflectarray configurations is presented in [4], including the use of thicker substrates with low permittivity, two-layer stacked patches having variable size, and aperture-coupled configuration with a phase tuning line. These structures are suitable for the implementation of small reflectarrays ( $D<50 \lambda_{0}$ at the operating frequency $f_{0}$ ) with wideband performances.

A further improvement in the bandwidth of aperturecoupled reflectarrays has been demonstrated by the authors in [5] through the reduction of the array lattice spacing. As a matter of fact, the use of closely spaced elements gives reflection phase curves with a smoother behavior with respect to the fixed tuning parameter as well as the frequency. As demonstrated in [6], this configuration can be effectively adopted for the design of broadband reflectarrays with reduced size. 


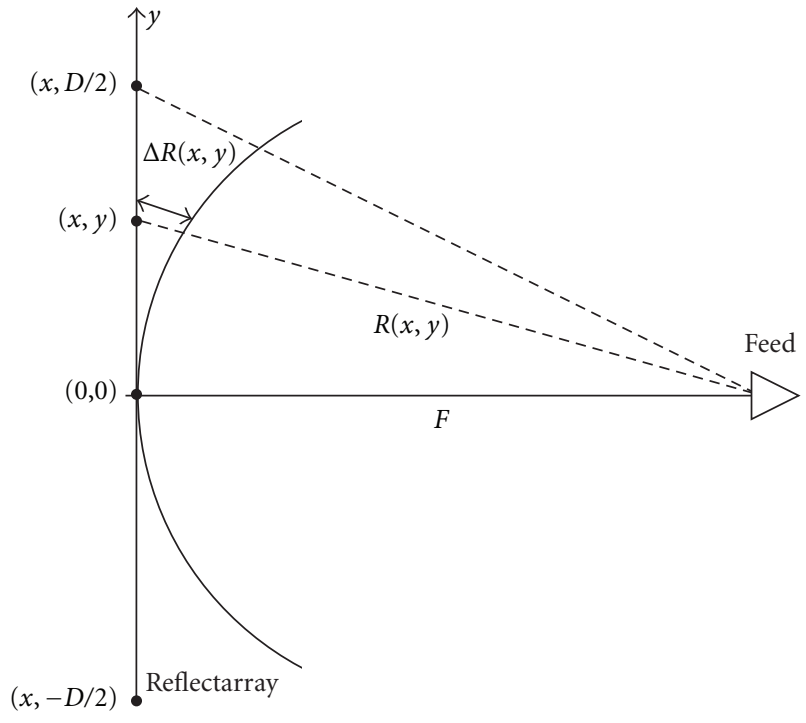

FIGURE 1: Reflectarray geometry.

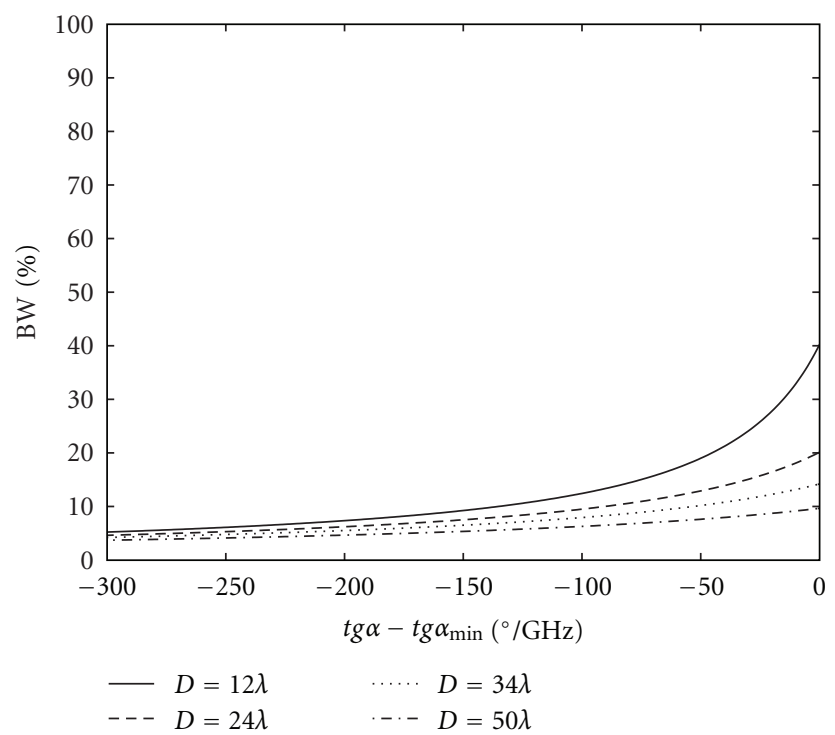

Figure 2: Percent bandwidth versus normalized phase curve slope for different array diameters $(F / D=0.5)$.

In this work, the bandwidth features of small reflectarrays are analyzed in terms of the phase errors due to the compensation mechanism for the spatial phase delays. A bandwidth estimation formula is derived which takes into account for the combined effects due to the overall antenna geometry and the frequency response of the single reflectarray element. The above formula provides an upper limit to the bandwidth achievable in the case of small reflectarrays with fixed geometry ( $D$ and $F / D$ values), giving a way to quantify the enhancement contribution due to elements with smoother phase curves. The adopted bandwidth evaluation approach is applied to the analysis of some reflectarrays prototypes, and it is shown that the relative $1 \mathrm{~dB}$ gain bandwidth value

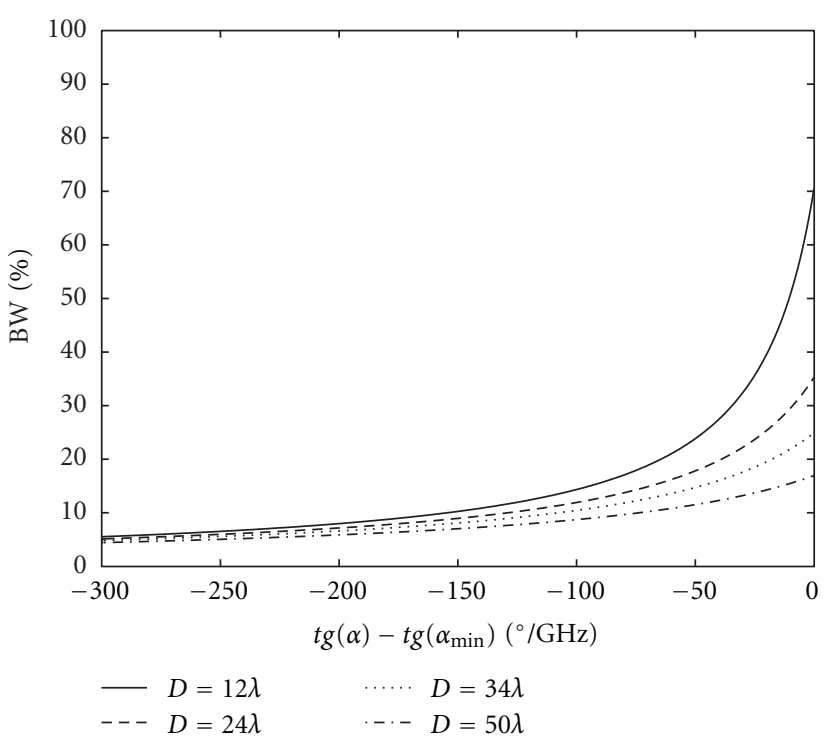

Figure 3: Percent bandwidth versus element phase curve slope for different array diameters $(F / D=1)$.

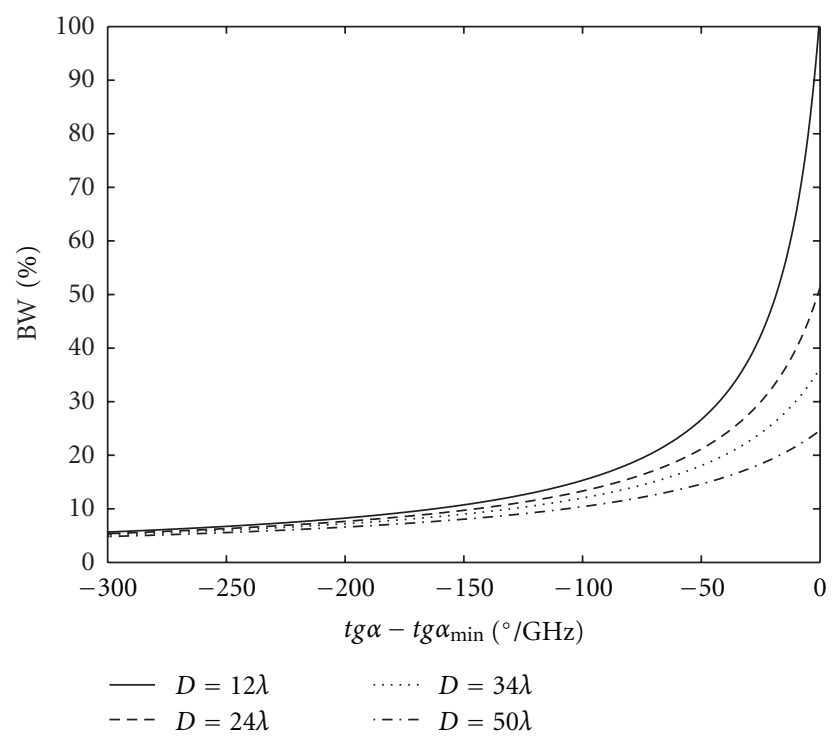

Figure 4: Percent bandwidth versus element phase curve slope for different array diameters $(F / D=1.5)$.

follows very well the behavior given by the proposed formula. As a matter of fact, other approaches existing in the literature $[7,8]$ for the bandwidth evaluation of reflectarrays assumed as validation reference the frequency range limiting the 1 $\mathrm{dB}$ gain of the antenna, that is, a zone where small or very negligible gain variations occur. In our case, the slope variations of the phase curves are taken into account to give a better upper bound with respect to the methods proposed in literature $[7,8]$. Although it is not demonstrated that an exact correlation exists between the proposed formula and the $1 \mathrm{~dB}$ gain bandwidth, the comparisons with this parameter are useful to make a qualitative analysis of the 


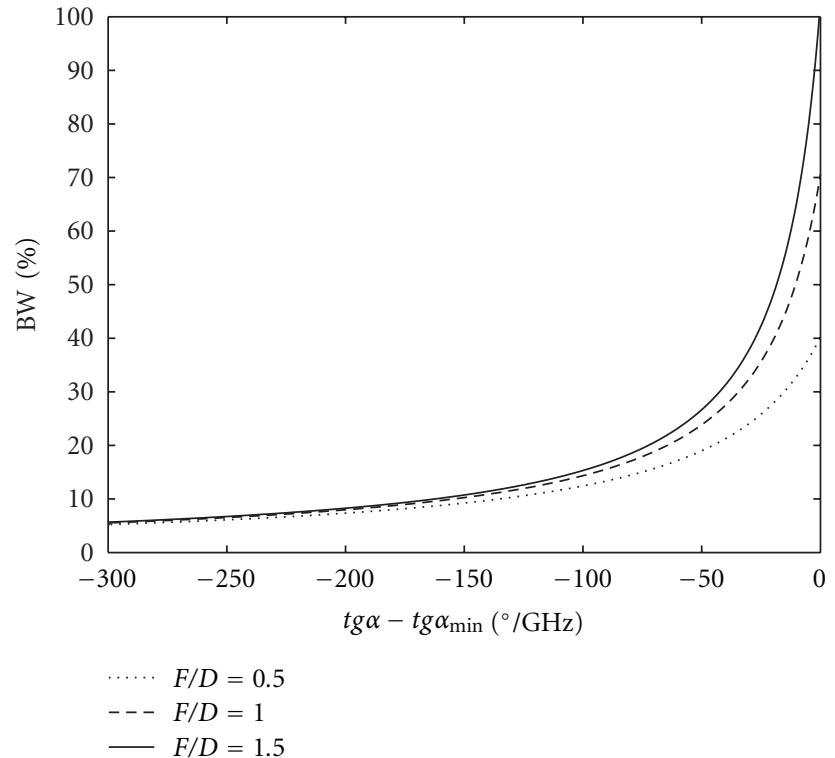

Figure 5: Percent bandwidth versus element phase curve slope for different $F / D$ ratios $(D=12.4 \lambda)$.

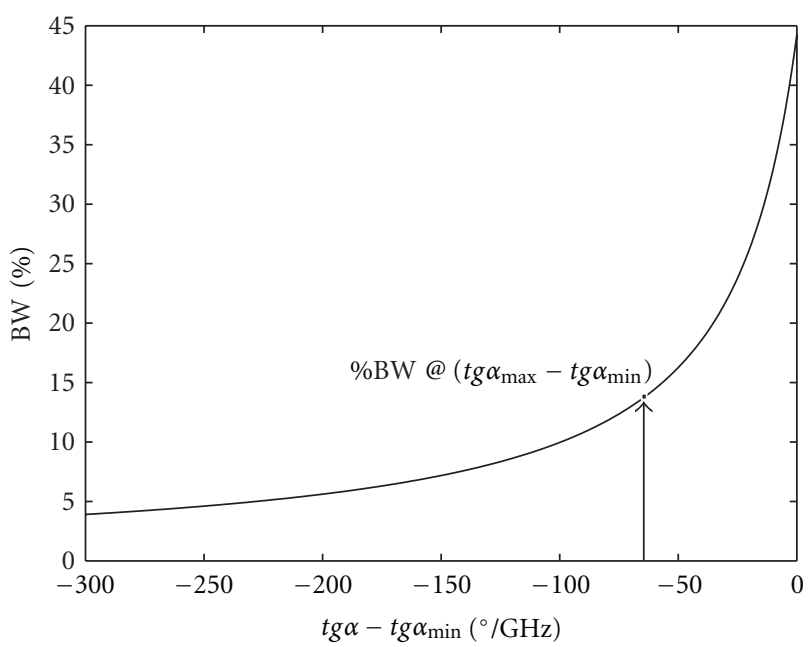

Figure 6: Percent bandwidth estimation for a $28 \mathrm{GHz}$ reflectarray $\left(D=14 \lambda_{0}-F / D=0.69\right)$ at a fixed phase curve slope.

reflectarray bandwidth improvement due to a proper choice of the single element configuration.

Furthermore, a $20 \mathrm{GHz}$ aperture-coupled element with a unit cell size smaller than $\lambda / 2$ is presented as broadband reflectarray radiator. The patch is printed on a $1 \mathrm{~mm}$-thick substrate of foam which also contributes to the element bandwidth enlargement. The synthesized element offers a phase curve with a very smooth behavior, essentially caused by the mutual coupling among the patches.

A significant bandwidth improvement (corresponding to a $1 \mathrm{~dB}$ gain bandwidth equal to $23 \%$ ) is demonstrated for a $12 \lambda$ diameter reflectarray operating at the frequency $f=$ $20 \mathrm{GHz}$.

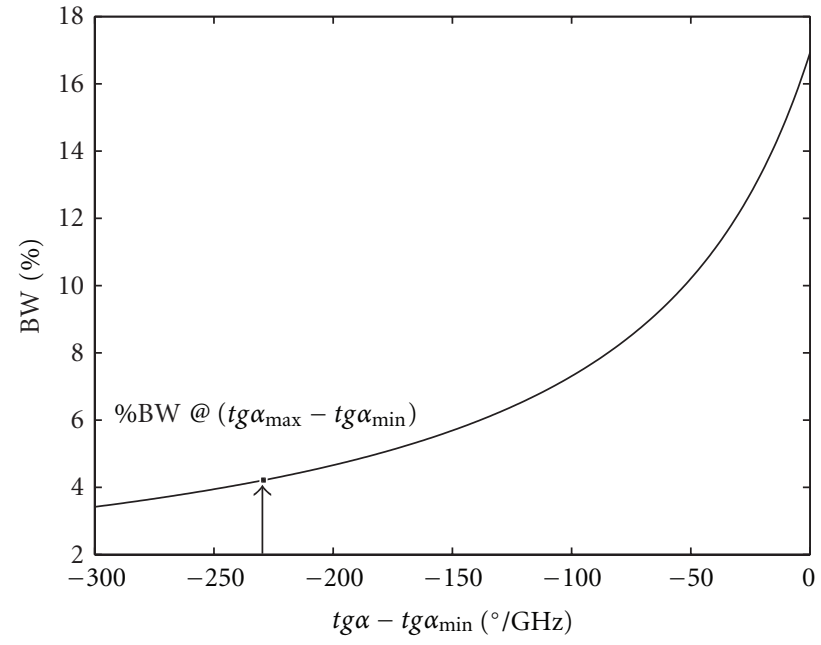

FIGURE 7: Percent bandwidth estimation for a $28 \mathrm{GHz}$ reflectarray $\left(D=21 \lambda_{0}-F / D=0.32\right)$ at a fixed phase curve slope.

\section{Bandwidth Estimation for Small Reflectarray Antennas}

The bandwidth of reflectarray antennas is strongly affected by the compensation mechanism for different phase delays in the paths from the feed to each array element. This effect is dominant in the case of large reflectarrays, so the development of techniques able to exactly compensate the differential phase delay in a large frequency range is required. Some of these techniques have been implemented in [1-4], giving appreciable bandwidth improvements. As demonstrated in $[4,7,8]$, the frequency dependence of the differential phase delay has a minor effect on the bandwidth of small reflectarrays. In this case, it is usually sufficient to adopt a wideband element configuration, in order to obtain a reflectarray with a relative large bandwidth. However, also for small aperture reflectarrays, the antenna frequency behavior must be optimized by considering the combined effect due to the bandwidth of the single element as well as the frequency dependence of the phase delay in the signal incident on each array element. Infact, the reflectarray geometry features, namely, the antenna diameter $D$ and the focal distance $F$, restrict the achievable bandwidth below an upper bound level $[7,8]$ that cannot be increased by the adoption of an element structure with wider bandwidth behavior. In order to demonstrate the above assertion, we consider the geometry of a center-fed reflectarray depicted in Figure 1. The feed antenna produces a spherical wave impinging on the reflectarray aperture. The path difference between the focal distance $F$ and each ray departing from the feed up to the generic element position $(x, y)$ is equal to the following:

$$
\Delta R(x, y)=\sqrt{F^{2}+x^{2}+y^{2}}-F
$$




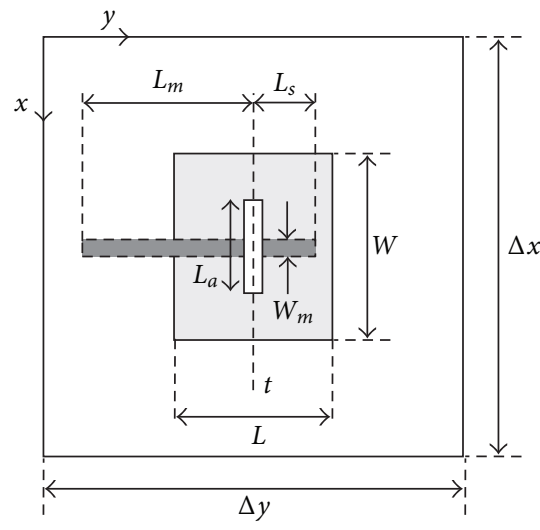

(a)

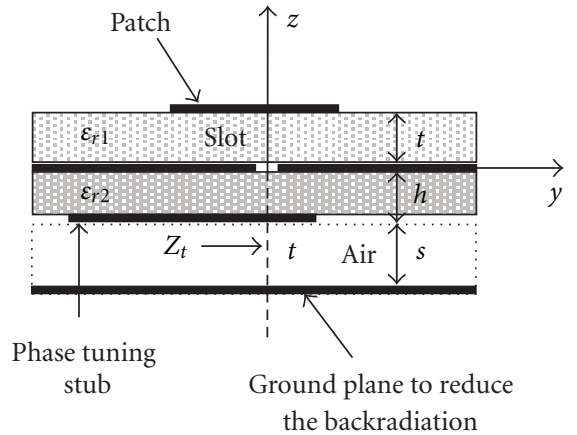

(b)

FIGURE 8: Aperture-coupled reflectarray unit cell—(a) top view and (b) side view.

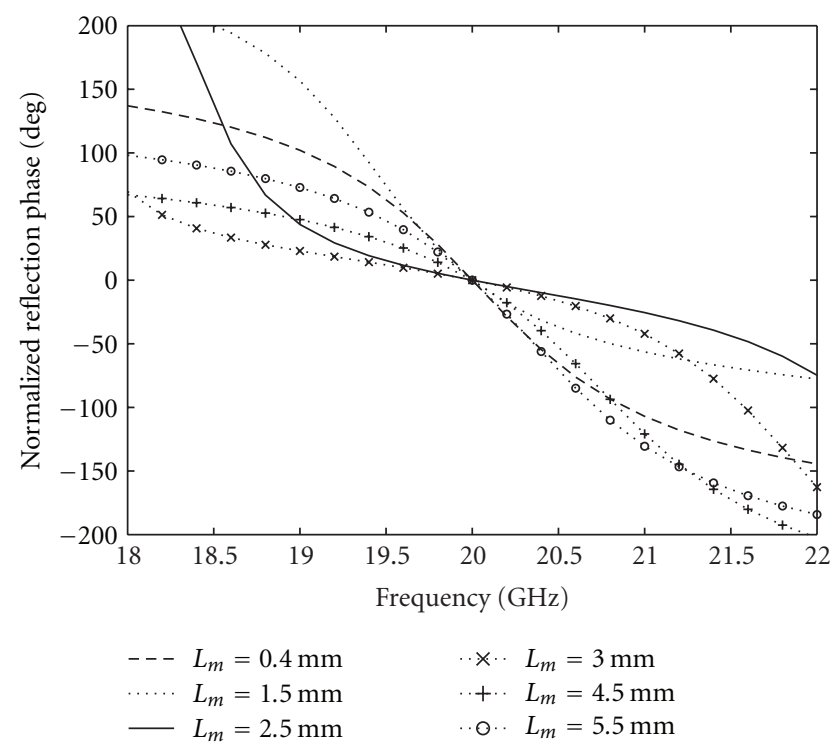

(a)

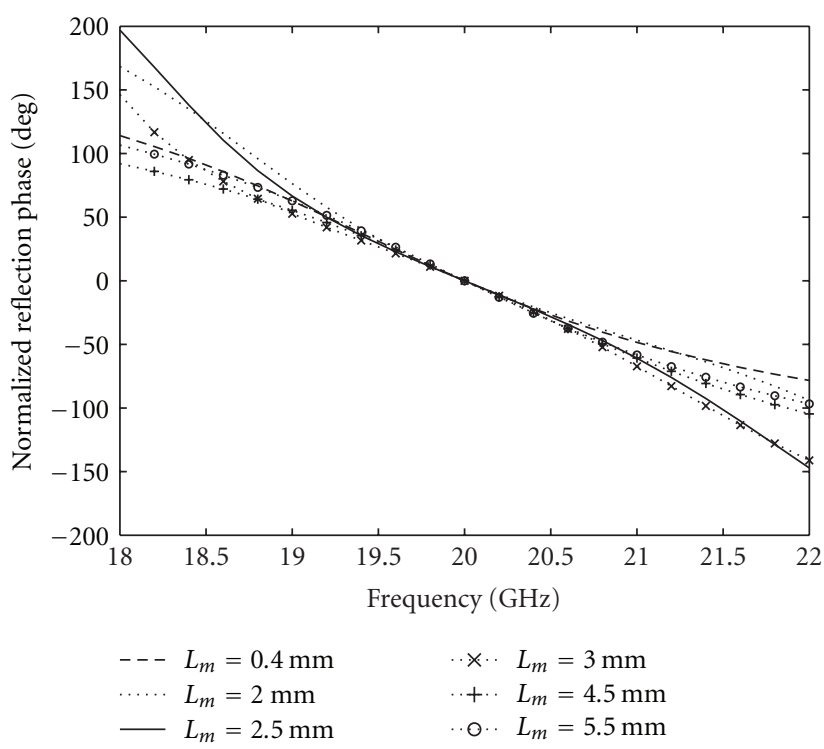

(b)

FIGURE 9: Calculated phase curves against frequency for various tuning line length-(a) $\Delta x=\Delta y=0.6 \lambda$; (b) $\Delta x=\Delta y=0.4 \lambda$.

A differential phase delay proportional to the above distance is imposed on each array position, giving the following incident phase:

$$
\phi_{\text {inc }}(x, y, f)=-\frac{2 \pi}{c_{0}} f\left(\sqrt{F^{2}+x^{2}+y^{2}}-F\right)
$$

where $c_{0}$ is the light velocity in vacuum.

Each reflectarray element must be designed in order to compensate for the phase delay (2) and to steer the main beam along a desired direction, at a given frequency $f_{0}$ [9].

For simplicity, the case of a broadside reflectarray is considered, for which the compensating phase to be introduced by the array elements is given as

$$
\phi_{\text {refl }}\left(x, y, f_{0}\right)=\frac{2 \pi}{c_{0}} f_{0}\left(\sqrt{F^{2}+x^{2}+y^{2}}-F\right)
$$

or

$$
\phi_{\text {refl }}\left(x, y, f_{0}\right)=-\frac{2 \pi}{c_{0}} f_{0}\left(\sqrt{F^{2}+\frac{D^{2}}{4}}-\sqrt{F^{2}+x^{2}+y^{2}}\right),
$$

according to the assumed phase normalization positions in the array grid, which are, respectively, the central element with coordinates $(x, y)=(0,0)$ and the elements onto the rim of the array, namely, $(x, y)=( \pm D / 2,0)$ or $(x, y)=$ $(0, \pm D / 2)$.

This phase compensation approach guarantees the fulfillment of the imposed reflectarray design constraints only at the operating frequency $f_{0}$. Expression (2) shows that the phase of the signal incident on each element is a function of the frequency $f$, thus a wideband operation mode can be obtained only if the adopted reflectarray elements are able 


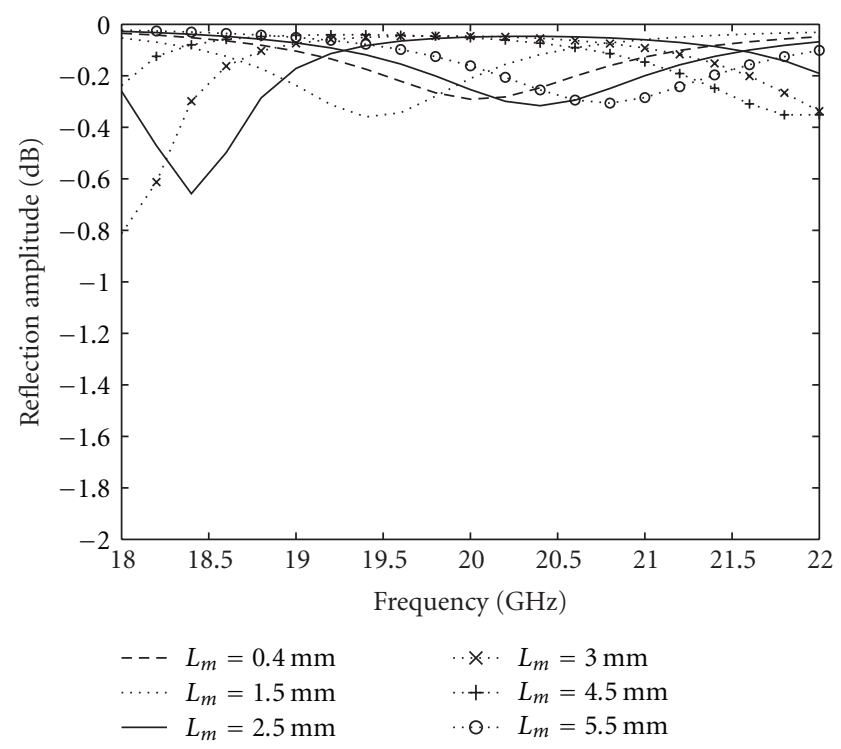

(a)

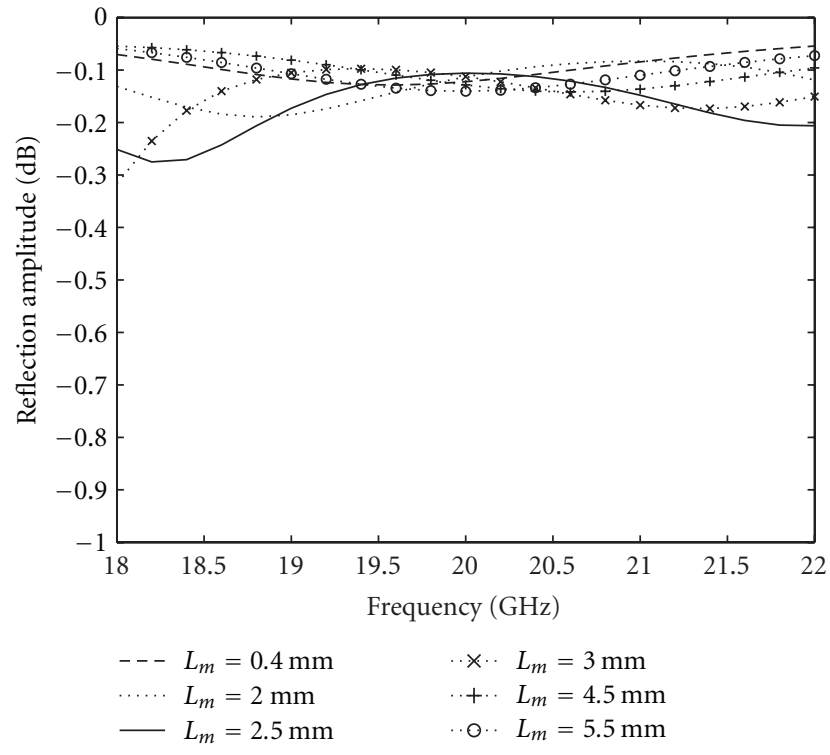

(b)

Figure 10: Calculated reflection amplitude against frequency for various tuning line length- (a) $\Delta x=\Delta y=0.6 \lambda$; (b) $\Delta x=\Delta y=0.4 \lambda$.

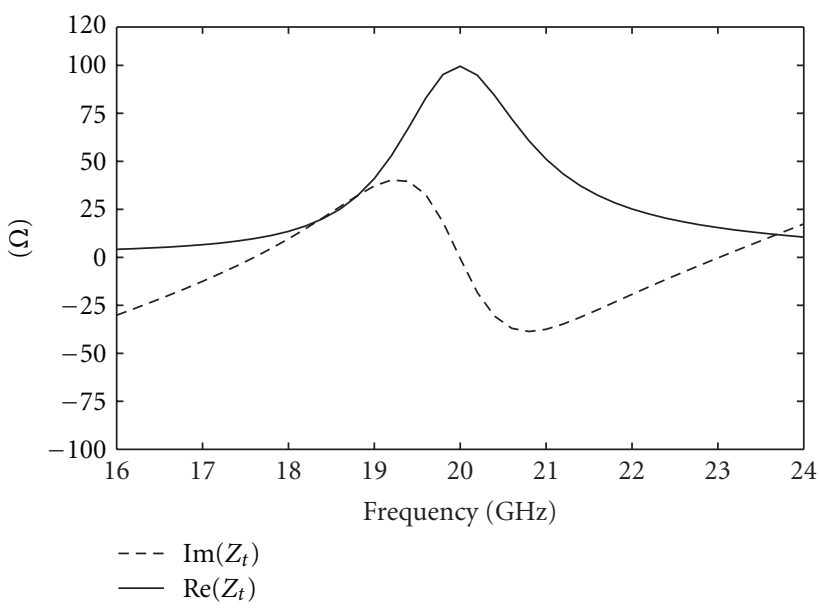

(a)

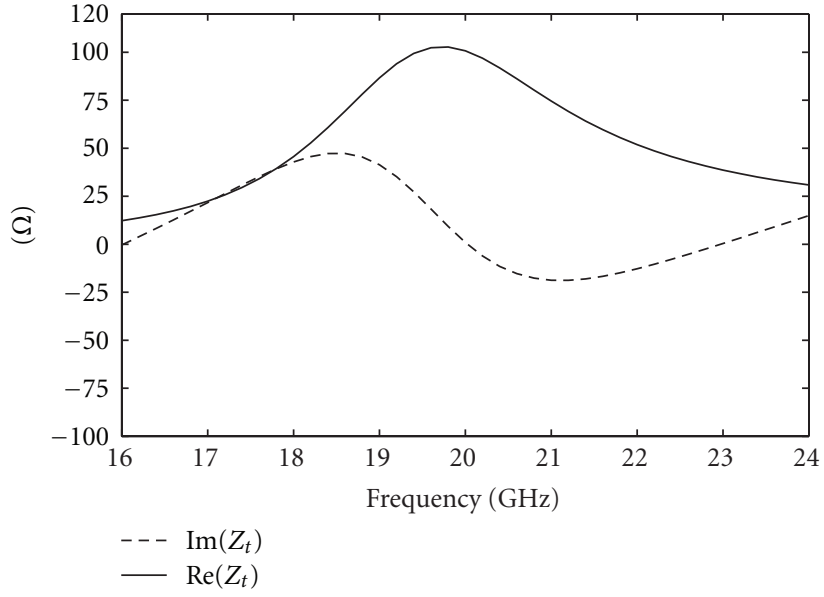

(b)

FIgURE 11: Simulated input impedance $Z_{t}$-(a) $\Delta x=\Delta y=0.6 \lambda$; (b) $\Delta x=\Delta y=0.4 \lambda$.

to exactly compensate the phase delay (2) in a rather wide neighborhood of $f_{0}$.

A further point to be considered is that the phase reflected by each reflectarray element is itself frequencydependent, that is, $\phi_{\text {refl }}(x, y, f)$. Its behavior is strictly related to the nature of the considered compensation technique, (e.g., variable size patches, aperture-coupled patches with variable lines, etc.) and, usually, it does not match the condition imposed by the frequency compensation of the incident phase (2). This last condition, infact, would require a linear behavior of $\phi_{\text {refl }}(x, y, f)$ with respect to the frequency, with a slope equal to the opposite value of the frequency derivative of (2). This means that the phase of the field reflected by the elements should have a frequency dependence with slightly different slopes for each position of the array grid [8]. It is very difficult to achieve this goal with the commonly used reflectarray configurations. In fact, they are not able to separately control the compensating phase value at the operating frequency $f_{0}$, given by $(3)$, and the rate of phase change with respect to the frequency $f$, given by the derivative $(\partial / \partial f) \phi_{\text {refl }}(x, y, f)$. Reflectarray configurations, based on the use of a single-phase tuning parameter, have a fixed frequency dependence for each achievable phase delay at the design frequency $f_{0}$.

Furthermore, the reflection phase curves of a broadband reflectarray element are almost parallel in a broad frequency range. This means that the slope of the reflection phase curves for a fixed reflectarray configuration can be assumed 


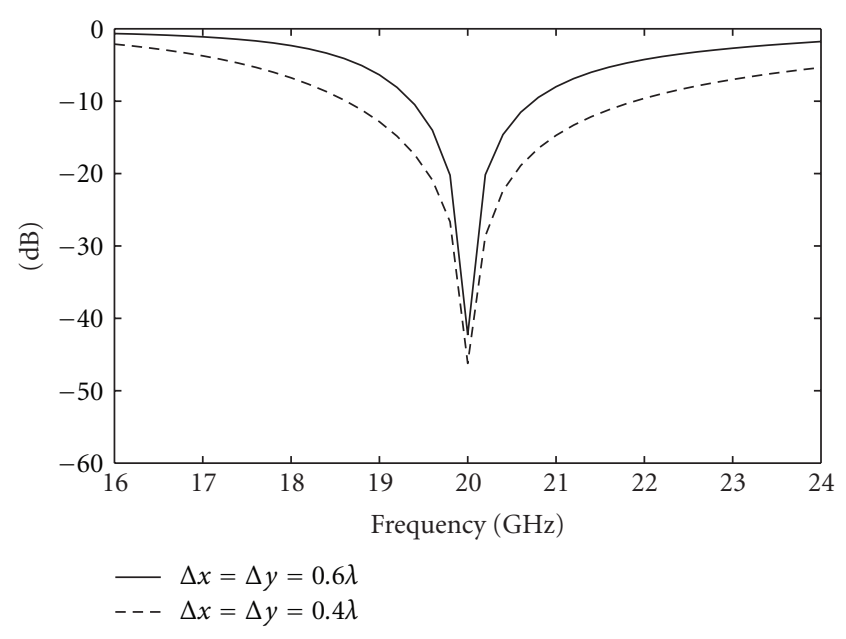

Figure 12: Simulated return loss.

constant with respect to the tuning parameter values. Thus, unless to develop reflectarray configurations offering more than one tuning parameter, in order to control both the required phase delay and the slope of the reflection phase curve, the bandwidth of this type of antenna cannot be optimized by the proper allocation of the elements in the array grid, according to the phase change rate requirements imposed by the derivative of expression (2).

Established that the incident phase (2) cannot be compensated for all frequencies, a phase error will occur, thus limiting the operating bandwidth of reflectarray antennas. In particular, the phase error increases when the operating frequency departs from the design frequency $f_{0}$, so introducing a reduction in the reflectarray gain bandwidth as well as a distortion of the synthesized radiation pattern.

The way to enlarge the bandwidth of a small reflectarray basically consists of the choice of an element configuration with a very slow frequency variation. However, the amount of achievable improvements by the adoption of phase curves with small slopes is limited by the antenna geometry. This fact is demonstrated below, by computing a new bandwidth estimation formula, like those presented in $[7,8]$, including also the dependence from the slope of the considered reflection phase curves. At this purpose, the frequency variation of the total phase obtained on the array surface is considered as follows:

$$
\phi_{\text {tot }}(x, y, f)=\phi_{\text {inc }}(x, y, f)+\phi_{\text {refl }}(x, y, f)
$$

with

$$
\phi_{\text {refl }}(x, y, f)=\phi_{\text {refl }}\left(x, y, f_{0}\right)+\operatorname{tg} \alpha\left(f-f_{0}\right),
$$

where $\operatorname{tg} \alpha$ is the phase variation rate versus frequency of the phase curve relative to the reflectarray elements. In general, $\operatorname{tg} \alpha$ varies with the tuning parameter, then it is a function of the location $(x, y)$. Furthermore, expression (6) assumes that the phase curves have a linear frequency dependence, but it is true only within a neighborhood of the frequency $f_{0}$, in accordance with the bandwidth performances of the adopted element configuration.
When the frequency varies from $f_{1}$ to $f_{2}$, with $f_{1}<f_{0}<$ $f_{2}$, the maximum phase change at each element position is given as:

$$
\Delta \phi_{\text {tot }}(x, y)=\left(f_{1}-f_{2}\right)\left[\operatorname{tg} \alpha-\frac{2 \pi}{c_{0}}\left(\sqrt{F^{2}+x^{2}+y^{2}}-F\right)\right] .
$$

In the case of broadband elements, characterized by phase curves with a strong similar slope for the various tuning parameter values, the phase error contribution in (7), due to the frequency dependence of the single radiator, can be considered identical in each location $(x, y)$ of the array, thus it can be omitted. This condition is equivalent to the assumption made in $[7,8]$, where a bandwidth estimation formula is computed by neglecting the frequency dependence of the phase reflected by the single reflectarray element, that is, $\operatorname{tg} \alpha=0^{\circ} / \mathrm{GHz}$. Thus, in this case the achievable maximum bandwidth is substantially imposed by the antenna geometry.

In the other cases, namely, those concerning relatively narrowband elements, or broadband elements with phase curves not so parallel, the phase curves slope varies within a certain range, namely $\operatorname{tg} \alpha \in\left(\operatorname{tg} \alpha_{\min } \div \operatorname{tg} \alpha_{\max }\right)$. In this latter case, the bandwidth could result to be much lower than the upper bound value computed in $[7,8]$. In particular, the wider is the slope variation range, the lower is the bandwidth upper bound level imposed in conjunction with the antenna geometry. At this purpose, the formula presented in [7] is changed in order to take into account the effects of the slope variation in the phase design curves associated to the adopted reflectarray elements.

Assuming a $360^{\circ}$ phase error at the edge of the aperture due to the considered frequency excursion, just like in [7], the percentage bandwidth $\mathrm{BW}=\left(f_{2}-f_{1}\right) / f_{0} \%$ can be evaluated as follows:

$$
\begin{aligned}
\mathrm{BW}= & \frac{-2 \pi}{f_{0}\left[\left(\operatorname{tg}(\alpha)-\operatorname{tg}\left(\alpha_{\min }\right)\right)-\left(2 \pi / c_{0}\right)\left(\sqrt{F^{2}+\left(D^{2} / 4\right)}-F\right)\right]} \\
& \times 100,
\end{aligned}
$$

in which the coordinates $(x, y)$ are fixed to the values corresponding to the edge aperture elements, that is, $( \pm D / 2,0)$ or $(0, \pm D / 2)$. Furthermore, the phase error $(7)$ is normalized with respect to the phase error contribution due to the minimum phase curve slope $\operatorname{tg} \alpha_{\text {min }}$, which is identical for each location $(x, y)$.

As follows, expression (8) is considered for the estimation of the phase curves slope effect on the reflectarray bandwidth, for different values of the antenna geometrical parameters.

Figure 2 shows the behavior of the percentage bandwidth versus the normalized phase change rate $\operatorname{tg}(\alpha)-\operatorname{tg}\left(\alpha_{\min }\right)$, for different values of the reflectarray diameter. The $F / D$ ratio is fixed to a value equal to 0.5 . It can be observed that BW decreases rapidly for higher phase change rates. Furthermore, for aperture diameters above the $40 \lambda$ value, the bandwidth is less dependent from the element phase curve 


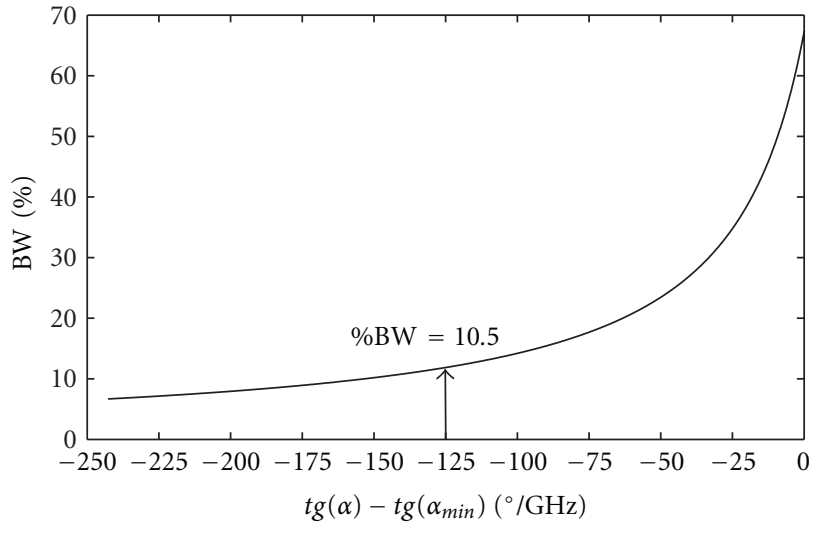

(a)

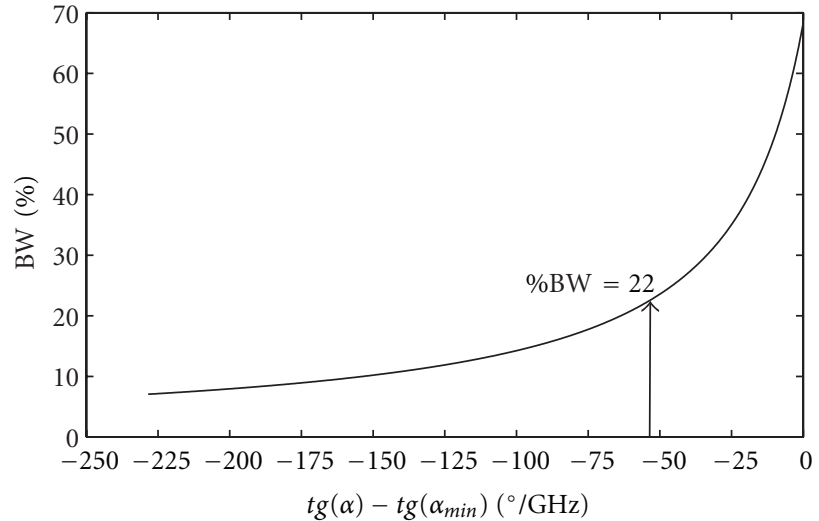

(b)

Figure 13: Percent bandwidth estimation $\left(D=12.4 \lambda_{0}-F / D=1\right)-(\mathrm{a}) \Delta x=\Delta y=0.6 \lambda$; (b) $\Delta x=\Delta y=0.4 \lambda$.

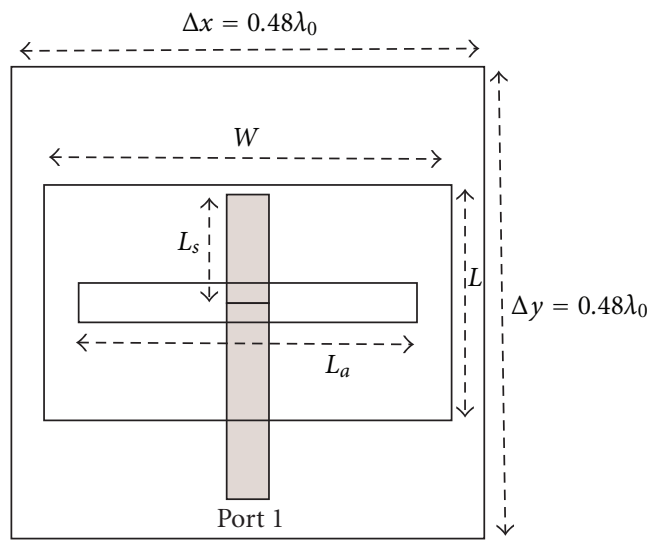

(a)

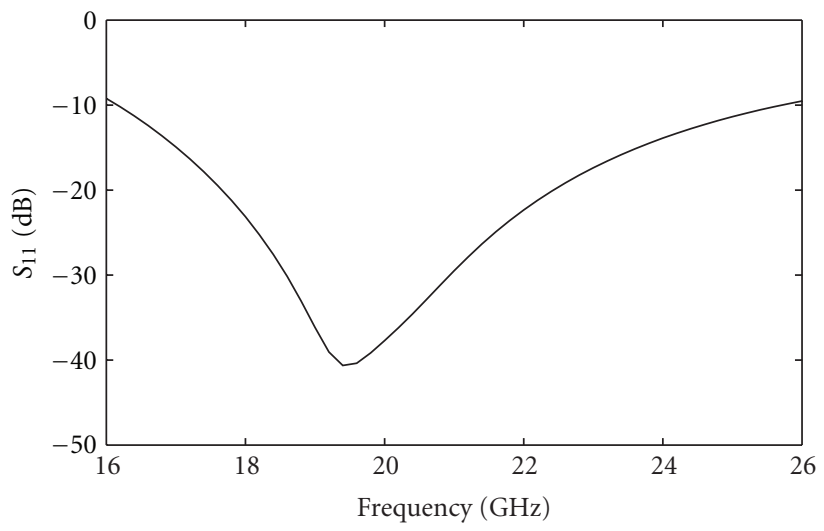

(b)

Figure 14: Aperture-coupled reflectarray unit cell—(a) top view and (b) simulated return loss at port 1.

slope. Similar considerations can be made for the graphs reported under Figures 3 and 4, illustrating the percentage bandwidth of a reflectarray with $F / D$ ratios, respectively equal to 1 and 1.5. Also in these cases different values of the array diameter are considered. From the comparison of the three plots (Figures 2-4), it can be observed that the bandwidth increases for higher $F / D$ ratios, but this trend is more pronounced for smaller array diameters. In every cases, it can be noted that a normalized phase change rate below the $-150^{\circ} / \mathrm{GHz}$ value, makes the percentage bandwidth independent from the antenna geometry. As a matter of fact, in this case it is dominant the effect of the intrinsic narroband behavior of the single radiator.

A small variation in the normalized phase curve slope within the $[-50 \div 0]^{\circ} / \mathrm{GHz}$ range may give a very large change in the estimated bandwidth value. This observation can be better appreciated in Figure 5, where the curves obtained for a $12.4 \lambda$ aperture diameter are compared for different $F / D$ ratios.

The bandwidth estimation, given by (8), is subject to certain changes due to the simplified assumption that the phase curves computed for different values of the tuning parameter are linear. However, the deviations from this ideal conditions are very small within the operating frequency range of broadband reflectarray element configurations. So, formula (8) gives a quite good trend of the bandwidth behavior for small reflectarray antennas.

In order to prove the effectiveness of the derived formula (8), this is adopted for the bandwidth estimation of two different reflectarrays with variable size elements, which have been presented in [9]. The antennas are characterized by the features reported in Table 1. In particular, the $\operatorname{tg}\left(\alpha_{\max }\right)-$ column refers to the slope of the reflection phase curves computed for the element length giving the resonance at the operating frequency $f_{0}$, corresponding to the maximum phase curve slope. Since both the considered reflectarray configurations are narrowband, the slope of the phase curves varies very rapidly with both the frequency and the element length within the range $\left(\operatorname{tg} \alpha_{\min } \div \operatorname{tg} \alpha_{\max }\right)$ specified in Table 1 .

In the same table, a comparison with previuosly published data is also reported, which are, respectively, referred to the measured reflectarray gain bandwidth, reported in 
TABLE 1: Bandwidth estimation values compared to previously published data.

\begin{tabular}{lccccccc}
\hline & \multicolumn{3}{c}{ Antenna parameters } & \multicolumn{3}{c}{ Bandwidth values } \\
$f_{0}(\mathrm{GHz})$ & $D(\mathrm{~m})$ & $F / D$ & $\operatorname{tg}\left(\alpha_{\max }\right)(\% / \mathrm{GHz})$ & $\operatorname{tg}\left(\alpha_{\min }\right)(\% / \mathrm{GHz})$ & $\begin{array}{c}\text { Percentage BW } \\
\text { measured [9] }\end{array}$ & $\begin{array}{c}\text { Percentage BW } \\
\text { calculated [8] }\end{array}$ & $\begin{array}{c}\text { Percentage BW } \\
\text { calculated }(8)\end{array}$ \\
\hline 28 & $0.152\left(\cong 14 \lambda_{0}\right)$ & 0.69 & -62 & -1.5 & 5 & 22.1 & 13 \\
28 & $0.23\left(\cong 21 \lambda_{0}\right)$ & 0.32 & -228 & -0.8 & 2 & 8.5 & 4 \\
\hline
\end{tabular}

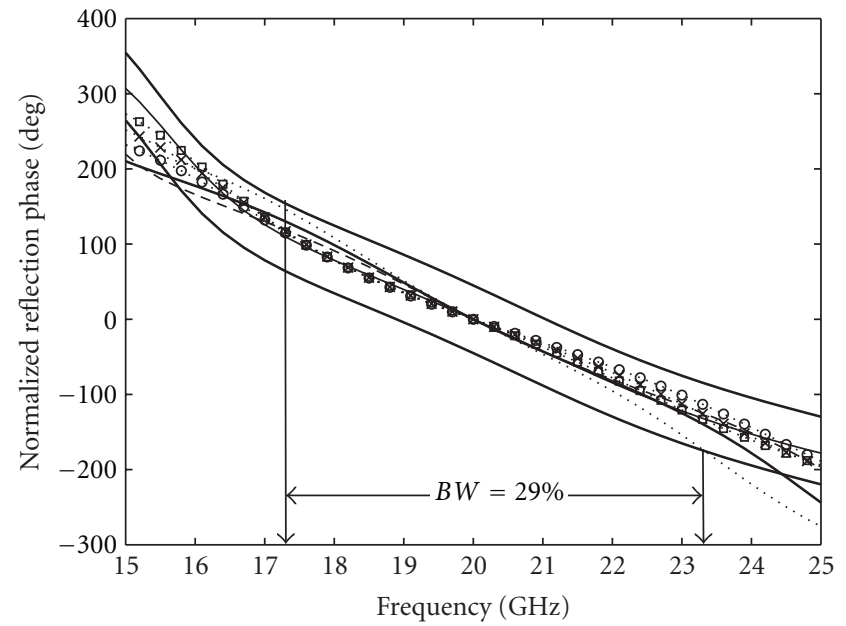

$$
\begin{aligned}
& \text { - Upper bound } \\
& \text { _ Lower bound } \\
& \text { ○. } L_{s}=0.75 \mathrm{~mm} \\
& \text { ․ } L_{s}=1.25 \mathrm{~mm} \\
& \text { ‥ } L_{s}=1.75 \mathrm{~mm} \\
& -L_{s}=2.75 \mathrm{~mm} \\
& \text { - - - } L_{s}=4.5 \mathrm{~mm} \\
& -L_{s}=5.25 \mathrm{~mm} \\
& L_{s}=6 \mathrm{~mm}
\end{aligned}
$$

FIGURE 15: Calculated phase curves against frequency for various tuning line length.

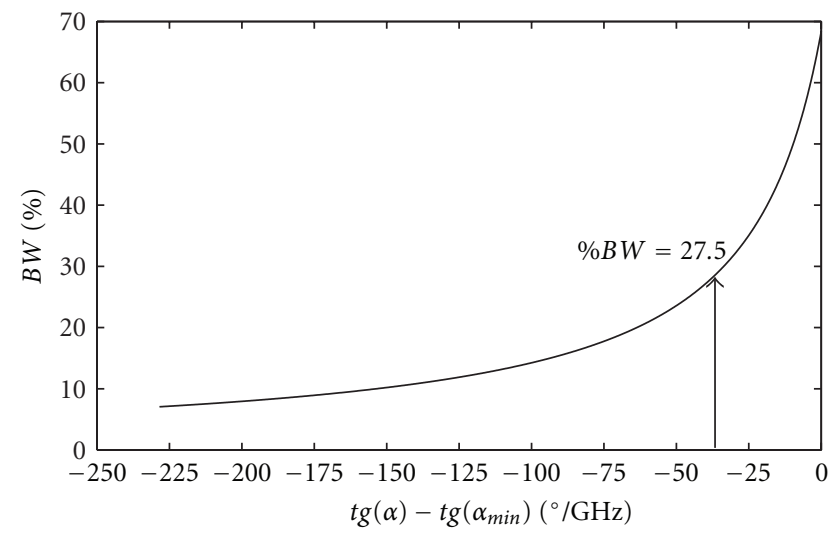

Figure 16: Percent bandwidth estimation $\left(D=12 \lambda_{0}, F / D=1\right.$, $\Delta x=\Delta y=0.48 \lambda)$.

[9], and the bandwidth calculated in [8]. The BW values estimated with the formula (8) are derived from the curves illustrated in Figures 6 and 7, by fixing the phase change rate $\operatorname{tg}(\alpha)$ to the values reported in Table 1. It can be observed that (8) gives a quite good estimation of the reflectarrays bandwidth which is closer to the measured values reported

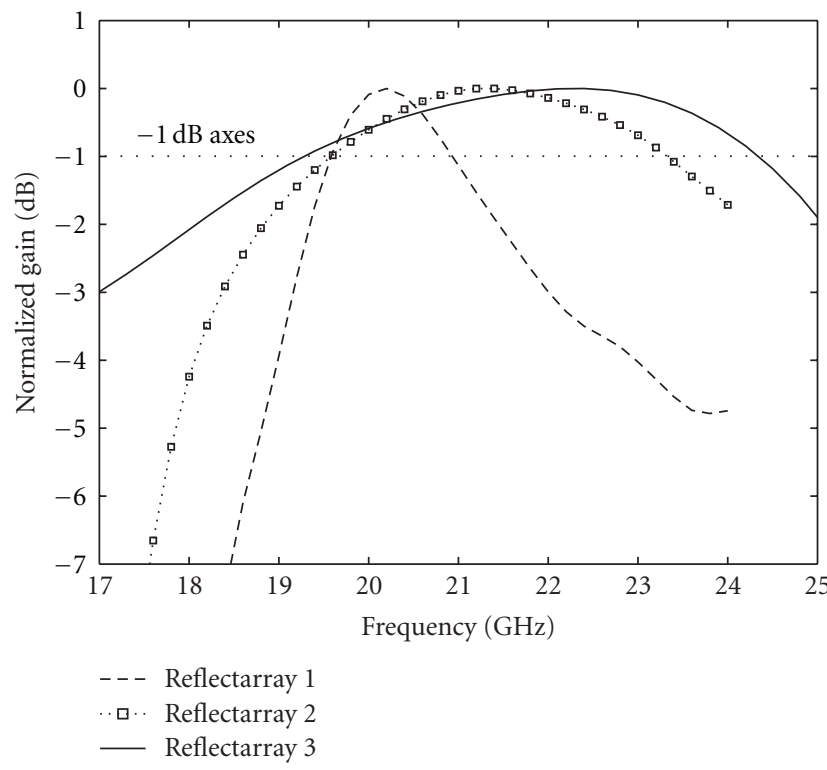

FIgURE 17: Comparison among the normalized gain patterns (broadside direction).

TABLE 2: Element stratification (reflectarray prototypes presented in $[6,7])$.

\begin{tabular}{lcc}
\hline Layer & Material & Thickness \\
\hline Patch & Copper & $35 \mu \mathrm{m}$ \\
d1 & Diclad $870\left(\varepsilon_{r 1}=2.33\right)$ & $t=0.762 \mathrm{~mm}$ \\
Ground plane with slot & Copper & $35 \mu \mathrm{m}$ \\
d2 & Diclad $870\left(\varepsilon_{r 2}=2.33\right)$ & $h=0.762 \mathrm{~mm}$ \\
Phasing line & Copper & $35 \mu \mathrm{m}$ \\
d3 & Air & $s=3.7 \mathrm{~mm}$ \\
Ground plane reducing & Copper & - \\
back radiation & & - \\
\hline
\end{tabular}

TABle 3: Antenna dimensions (reflectarray unitary cell equal to: $\Delta x=\Delta y=0.6 \lambda$ and $\Delta x=\Delta y=0.4 \lambda$.

\begin{tabular}{lcc}
\hline Layer & $\Delta x=\Delta y=0.6 \lambda$ & $\Delta x=\Delta y=0.4 \lambda$ \\
\hline Patch $(W \times L)$ & $4.9 \mathrm{~mm} \times 3.68 \mathrm{~mm}$ & $4.9 \mathrm{~mm} \times 3.2 \mathrm{~mm}$ \\
Slot $\left(W_{a} \times L_{a}\right)$ & $0.4 \mathrm{~mm} \times 3.25 \mathrm{~mm}$ & $0.4 \mathrm{~mm} \times 3.8 \mathrm{~mm}$ \\
Stub $\left(W_{m} \times L_{s}\right)$ & $0.65 \mathrm{~mm} \times 1.98 \mathrm{~mm}$ & $0.65 \mathrm{~mm} \times 2.1 \mathrm{~mm}$ \\
\hline
\end{tabular}

in [9]. Other parameters affect the measured $1 \mathrm{~dB}$ gain bandwidth, which are not considered into (8), such as the field pattern of the feed illuminating the array and the dielectric/conductor losses of the elements. However, the 
TABLE 4: Element stratification.

\begin{tabular}{lcc}
\hline Layer & Material & Thickness \\
\hline Covering dielectric & Diclad $870\left(\varepsilon_{r}=2.33\right)$ & $0.762 \mathrm{~mm}$ \\
Patch & Copper & $35 \mu \mathrm{m}$ \\
d1 & Foam $\left(\varepsilon_{r 1}=1.07\right)$ & $1 \mathrm{~mm}$ \\
Ground plane with slot & Copper & $35 \mu \mathrm{m}$ \\
d2 & Diclad $870\left(\varepsilon_{r 2}=2.33\right)$ & $0.762 \mathrm{~mm}$ \\
Phasing line & Copper & $35 \mu \mathrm{m}$ \\
d3 & Air & $3.7 \mathrm{~mm}$ \\
Ground plane reducing & Copper & - \\
back radiation & & \\
\hline
\end{tabular}

provided estimation formula, as discussed above, gives a closer upper bound to the effective measured bandwidth values, when compared to the existing approaches $[7,8]$.

\section{Broadband Aperture-Coupled Reflectarray Configurations with Reduced Interelement Spacing}

The analysis performed in the previous paragraph has confirmed the fact that the bandwidth of small reflectarrays is strongly affected by the slope variation of the single elements phase curves versus frequency. Furthermore, the reflectarray bandwidth depends on the extent of the frequency range within which the slope of overall phase curves can be considered identical. So, the bandwidth behavior of reflectarrays with moderate size is controlled by the combined effect of the single element bandwidth and the slope of the reflection phase curves. As a result, the choice of the single element configuration, satisfying the bandwidth requirements on the overall reflectarray antenna, must be done looking at the value of the reflected phase curve derivative versus the frequency. As demonstrated by the curves illustrated in Figures 3-5, in order to maximize the reflectarray bandwidth, the value of the curves slope should tend to zero or equivalently the curves must be almost parallel. Anyway, it must be fixed by taking into account the geometry of the antenna to be realized, such as the aperture diameter $D$ and the $F / D$ ratio.

In order to obtain a broader-band reflectarray with moderate size, the aperture-coupled configuration depicted in Figure 8, with a phase tuning stub slot-coupled to a rectangular patch, is optimized. The reflection phase is controlled by varying the length of the line section $L_{m}$, while all other antenna dimensions are fixed in order to match the aperture coupled patch with the variable length line.

This element is proposed as broadband reflectarray configuration $[4,10]$, because it offers smoother phase variations when compared to the standard reflectarray configuration with patches of different size. Further bandwidth improvements of the aperture-coupled configuration have been demonstrated in $[5,6]$, by reducing the spacing between adjacent elements in the array grid. These enhancements are principally due to the mutual coupling among the array elements which has the effects to reduce the slope of the phase curves versus frequency. In $[5,6]$, the comparison between two reflectarrays characterized by different grid spacing is reported. The two considered configurations are characterized by the same layers stratification reported under Table 2, but they have different cell dimensions that are, respectively, $\Delta x=\Delta y=0.6 \lambda_{0}$ and $\Delta x=\Delta y=0.4 \lambda_{0}$ at the operating frequency $f_{0}=20 \mathrm{GHz}$. The dimensions of the two synthesized reflectarray elements are reported under Table 3. The characteristic impedance of the tuning line is fixed to a value of $100 \Omega$. A wider band behavior has been demonstrated in [6] for the configuration with reduced spacing. Both the configurations have been analyzed with a full-wave code based on the infinite array approach and by assuming a normally incident plane wave.

For the sake of clarity, some results obtained in $[5,6]$ are reported below. In particular, Figures 9 and 10 show, respectively, the phase and the amplitude of the reflection coefficient computed for the two considered cases. The phase curves computed in the case of a $0.4 \lambda_{0}$ spacing show a smoother behavior which extends over a larger frequency range with respect to the case of a $0.6 \lambda_{0}$ cell. All the phase curves are normalized with respect to the central frequency $f_{0}$. The maximum slope $\operatorname{tg} \alpha_{\max }$ of the computed curves is evaluated to be equal to $-150^{\circ} / \mathrm{GHz}$ and $-88^{\circ} / \mathrm{GHz}$ for the $0.6 \lambda_{0}$ and the $0.4 \lambda_{0}$ unit cell size, respectively, while the minimum curve slope $\operatorname{tg} \alpha_{\min }$ is equal to $-25^{\circ} / \mathrm{GHz}$ and $-35^{\circ} / \mathrm{GHz}$, respectively. Similarly, the amplitude of the reflection coefficient (Figure 10) shows slower variations with respect to the frequency in the case of the $0.4 \lambda_{0}$ cell. Anyway, it can be observed that in both cases the total losses due to the dielectric and the conductors are very low. As a matter of fact, the reflection amplitude remains above the $-0.8 \mathrm{~dB}$ level. This means that the losses associated to the single radiator will cause a negligible reduction of the reflectarray antenna efficiency, which in turn will be principally affected by the spillover and the tapering losses [9].

The strong bandwidth improvement obtained in the case of the reduced cell size is determined by the coupling between the patches. In the case of slot-coupled configuration, such as in multiresonant antennas [11], this allows to increase the impedance bandwidth between the slot-coupled patches and the variable length lines. As it is well known, a reflectarray element well matched to the phasing stub in a given frequency band is able to provide the appropriate phasing in the whole band [4]. Figures 11 and 12 illustrate the input impedance and the reflection coefficient seen at the line section $t$ (see Figure 8). It is evident that a broadband impedance bandwidth is obtained when the patch is embedded into an infinite array with a more dense lattice.

In order to verify the bandwidth enhancement due to the adoption of reflectarray elements with smoother phase curves, the $1 \mathrm{~dB}$ gain bandwidth has been computed in [6] for few reflectarrays with different aperture diameters, based on the two differently spaced element configurations.

The normalized gain patterns against frequency is calculated as described in [9], by the superimposition of the spherical waves radiated by each unitary cell composing the 
TABLE 5: Designed reflectarrays features. (operating frequency $f_{0}=20 \mathrm{GHz} ; F / D$ ratio $=1$ ).

\begin{tabular}{|c|c|c|c|c|c|}
\hline Reflectarray configuration & $D$ & $\Delta \mathrm{x}$ & Patch substrate & $\Delta \operatorname{tg}(\alpha)\left({ }^{\circ} / \mathrm{GHz}\right)$ & 1-dB gain bandwidth \\
\hline 1 & $12.6 \lambda_{0}$ & $0.6 \lambda_{0}$ & $\begin{array}{c}\varepsilon_{r 1}=2.33 \\
t=0.762 \mathrm{~mm}\end{array}$ & -125 & $6.7 \%$ \\
\hline 2 & $12.4 \lambda_{0}$ & $0.4 \lambda_{0}$ & $\begin{array}{c}\varepsilon_{r 1}=2.33 \\
t=0.762 \mathrm{~mm}\end{array}$ & -53 & $17 \%$ \\
\hline 3 & $12 \lambda_{0}$ & $0.48 \lambda_{0}$ & $\begin{array}{c}\varepsilon_{r 1}=1.07 \\
t=1 \mathrm{~mm}\end{array}$ & -37 & $23 \%$ \\
\hline
\end{tabular}

antenna, which are proportional to the reflection coefficients illustrated in Figures 9 and 10. The field pattern of the feed is also taken into account and modeled by the expression $G_{f}(\theta, \varphi)=\cos ^{n}(\theta)$, as usually adopted in the literature [9].

A bandwidth improvement is demonstrated for all considered case when $\Delta x$ is reduced to $0.4 \lambda_{0}$. In particular, when $D \cong 12.4 \lambda_{0}$ and $F / D=1$, the gain bandwidth increases from the value of $6.7 \%\left(\Delta x=\Delta y=0.6 \lambda_{0}\right)$ to the value of $17 \%\left(\Delta x=\Delta y=0.6 \lambda_{0}\right)$. These bandwidth values follow enough the behavior provided by the estimation formula (8), as it can be observed in Figure 13.

In order to obtain a further improvement in the bandwidth of the considered reflectarray configuration, a $1 \mathrm{~mm}$ thick foam substrate is adopted as patch dielectric support, while a reduced unit-cell size equal to $0.48 \lambda_{0}$ is considered. The designed element is characterized by the layers stratification reported under Table 4, while its top view is illustrated in Figure 14(a). The dimensions of the synthesized structure are the following: $W=6.2 \mathrm{~mm}, L=$ $3.6 \mathrm{~mm}, L_{a}=5.15 \mathrm{~mm}, W_{a}=0.6 \mathrm{~mm}, L_{s}=1.65 \mathrm{~mm}$, and $W_{s}=0.65 \mathrm{~mm}$.

The element shows a very large bandwidth in terms of $-10 \mathrm{~dB}$ return loss at the input port 1 (Figure 14(b)), which extends from $16.2 \mathrm{GHz}$ to $25.8 \mathrm{GHz}$. However, this high bandwidth value, usually, does not exactly match the bandwidth of the reflectarray element evaluated with respect to the reflection curves behavior [4]. The bandwidth of the designed reflectarray element is calculated using the approach described in [6], based on the evaluation of the frequency range within which all the phase curves computed for different values of the tuning stub $L_{m}$ are almost parallel. Then, normalizing all the curves with respect to the central frequency $f_{0}$, the operating band is computed by considering the parallelism condition satisfied, apart from a margin error $\Delta \phi$ equal to $\pm 45^{\circ}$. This bandwidth evaluation approach, applied to the above reflectarray element, gives the results illustrated in Figure 15, where an upper and a lower bound curve are used to delimitate the operating frequency range. The two curves are computed as the summation of a fixed reference curve with the error $\Delta \phi$. As it can be observed, the resulting bandwidth is equal to the value of about $29 \%$, while the maximum curves slope $\operatorname{tg}\left(\alpha_{\max }\right)$ is equal to about $-67^{\circ} / \mathrm{GHz}$ and the minimum slope $\operatorname{tg}\left(\alpha_{\min }\right)$ is about $-30^{\circ} / \mathrm{GHz}$. Matching this last values with the bandwidth estimating curve in Figure 16, a BW equal to $27.5 \%$ is obtained for a reflectarray with $D=12 \lambda_{0}$ and $F / D=1$.
The designed aperture-coupled element is adopted for the design of a reflectarray antenna operating at the frequency $f_{0}=20 \mathrm{GHz}$. The antenna is characterized by a circular reflecting surface with a diameter $D$ of about $12 \lambda_{0}$. The synthesized reflectarray has a unit cell size equal to $0.48 \lambda_{0} \times 0.48 \lambda_{0}$, with an overall number of 438 elements. The bandwidth behavior of the antenna is compared with the results obtained in [6] for two reflectarrays operating at the same frequency $f_{0}$ and having $D \cong 12.4 \lambda_{0}$. For all considered reflectarrays, the $F / D$ ratio is fixed to 1 , while the power radiation pattern of the feed is modeled by the expression $G_{f}(\theta, \varphi)=\cos ^{n}(\theta)$, with $n=10$.

A synthesis algorithm based on the iterative projection method $[12,13]$ is applied to compensate for the path delay on each reflecting element, so concentrating the impinging energy in the broadside direction.

Table 5 summarizes the principal features of all considered reflectarray antennas. The same table reports the $1 \mathrm{~dB}$ gain bandwidth value calculated for each antenna prototype. The comparison among the three normalized gain patterns is illustrated in Figure 17. It can be observed a significant enlargement in the bandwidth of the reflectarray based on the aperture-coupled element proposed in this work. As previously discussed, this wider band behavior is due to the smaller slope of the almost parallel reflection phase curves related to the designed reflectarray element.

\section{Conclusion}

A bandwidth estimation criterion for the reflectarray bandwidth has been derived, taking into account the combined effect due to the antenna geometry and the frequency dependence of the phase reflected by each reflectarray element. The formula has been adopted for the estimation of the maximum achievable bandwidth for some closely spaced aperture-coupled reflectarrays, showing a good agreement with the calculated $1 \mathrm{~dB}$ gain bandwidth for each considered case. A significant bandwidth enlargement has been demonstrated by reducing the phase curve slopes through a proper tuning of the coupling between the closely spaced elements [14]. Concerning Future developments, the application of the proposed method to broadband millimeter-wave $[15,16]$ passive and/or active [17-19] reflectarrays on innovative dielectric materials [20] will be considered, and experimental characterizations will be provided also by the adoption of innovative near-field [21-23] and openresonator [24] techniques. 


\section{References}

[1] J. Huang, "Analysis of a microstrip reflectarray antenna for microspacecraft applications," TDA Progress Report, 1995.

[2] J. A. Encinar and J. A. Zornoza, "Broadband design of threelayer printed reflectarrays," IEEE Transactions on Antennas and Propagation, vol. 51, no. 7, pp. 1662-1664, 2003.

[3] A. Roederer, "Reflector antenna comprising a plurality of panels," US6411255, 2002.

[4] J. Huang and J. A. Encinar, Reflectarray Antennas, IEEE Press, Wiley-Interscience, 2008.

[5] S. Costanzo, F. Venneri, and G. Di Massa, "Bandwidth enhancement of aperture-coupled reflectarrays," Electronics Letters, vol. 42, no. 23, pp. 1320-1321, 2006.

[6] F. Venneri, S. Costanzo, G. Di Massa, and G. D. Amendola, "Aperture-coupled reflectarrays with enhanced bandwidth features," Journal of Electromagnetic Waves and Applications, vol. 22, no. 11-12, pp. 1527-1537, 2008.

[7] D. M. Pozar, "Bandwidth of reflectarrays," Electronics Letters, vol. 39, no. 21, pp. 1490-1491, 2003.

[8] M. E. Bialkowski and K. H. Saydmarie, "Bandwidth cinsiderations for a microstrip reflectarray," Progress In Electromagnetics Research B, vol. 3, pp. 173-187, 2008.

[9] D. M. Pozar, S. D. Targonski, and H. D. Syrigos, "Design of millimeter wave microstrip reflectarrays," IEEE Transactions on Antennas and Propagation, vol. 45, no. 2, pp. 287-296, 1997.

[10] F. Venneri, S. Costanzo, and G. Di Massa, "Transmission line analysis of aperture-coupled reflectarrays," Progress in Electromagnetics Research C, vol. 4, pp. 1-12, 2008.

[11] K. L. Wong, Compact and Broadband Microstrip Antennas, John Wiley \& Sons, 2002.

[12] F. Venneri, S. Costanzo, G. Di Massa, and G. Angiulli, "An improved synthesis algorithm for reflectarrays design," IEEE Antennas and Wireless Propagation Letters, vol. 4, no. 1, pp. 258-261, 2005.

[13] S. Costanzo, F. Venneri, G. Di Massa, and G. Angiulli, "Synthesis of microstrip reflectarrays as planar scatterers for SAR interferometry," Electronics Letters, vol. 39, no. 3, pp. 266267, 2003.

[14] F. Caminita, S. Costanzo, G. Di Massa et al., "Reduction of patch antenna coupling by using a compact EBG formed by shorted strips with interlocked branch-stubs," IEEE Antennas and Wireless Propagation Letters, vol. 8, pp. 811-814, 2009.

[15] S. Costanzo, I. Venneri, G. Di Massa, and G. Arriendola, "Hybrid array antenna for broadband millimeter-wave applications," Progress In Electromagnetics Research-PIER, vol. 83, pp. 173-183, 2008.

[16] S. Costanzo, "Synthesis of multi-step coplanarwaveguidetomicrostrip transition," Progress In Electromagnetics ResearchPIER, vol. 113, pp. 111-126, 2011.

[17] F. Venneri, S. Costanzo, and G. Di Massa, "Reconfigurable aperturecoupled reflectarray element tuned by single varactor diode," Electronics Letters, vol. 48, no. 2, pp. 68-69, 2012.

[18] F. Venneri, S. Costanzo, G. Di Massa et al., "Beam-scanning reflectarray based on a single varactor-tuned element," International Journal of Antennas and Propagation, vol. 2012, Article ID 290285, 5 pages, 2012.

[19] F. Venneri, S. Costanzo, G. Di Massa, P. Corsonello, and M. Salzano, "Design of a reconfigurable reflectarray based on a varactor tuned element," in Proceedings of the 6th European Conference on Antennas and Propagation (EUCAP '12), pp. 2628-2631, Prague, Czech Republic, March 2012.
[20] S. Costanzo, I. Venneri, G. Di Massa, and A. Borgia, "Benzocyclobutene as substrate material for planar millimeterwave structures: dielectric characterization and application," Journal of Infrared, Millimeter and Terahertz Waves, vol. 31, no. 1, pp. 66-77, 2010.

[21] S. Costanzo and G. Di Massa, "Far-field reconstruction from phaseless near-field data on a cylindrical helix," Journal of Electromagnetic Waves and Applications, vol. 18, no. 8, pp. 1057-1071, 2004.

[22] S. Costanzo and G. Di Massa, "Direct far-field computation from bi-polar near-field samples," Journal of Electromagnetic Waves and Applications, vol. 20, no. 9, pp. 1137-1148, 2006.

[23] S. Costanzo and G. Di Massa, "Near-field to far-field transformation with planar spiral scanning," Progress In Electromagnetics Research-PIER, vol. 73, pp. 49-59, 2007.

[24] G. Di Massa, S. Costanzo, and O. H. Moreno, "Open resonator system for reflectarray elements characterization," International Journal of Antennas and Propagation, vol. 2012, Article ID 912809, 7 pages, 2012. 

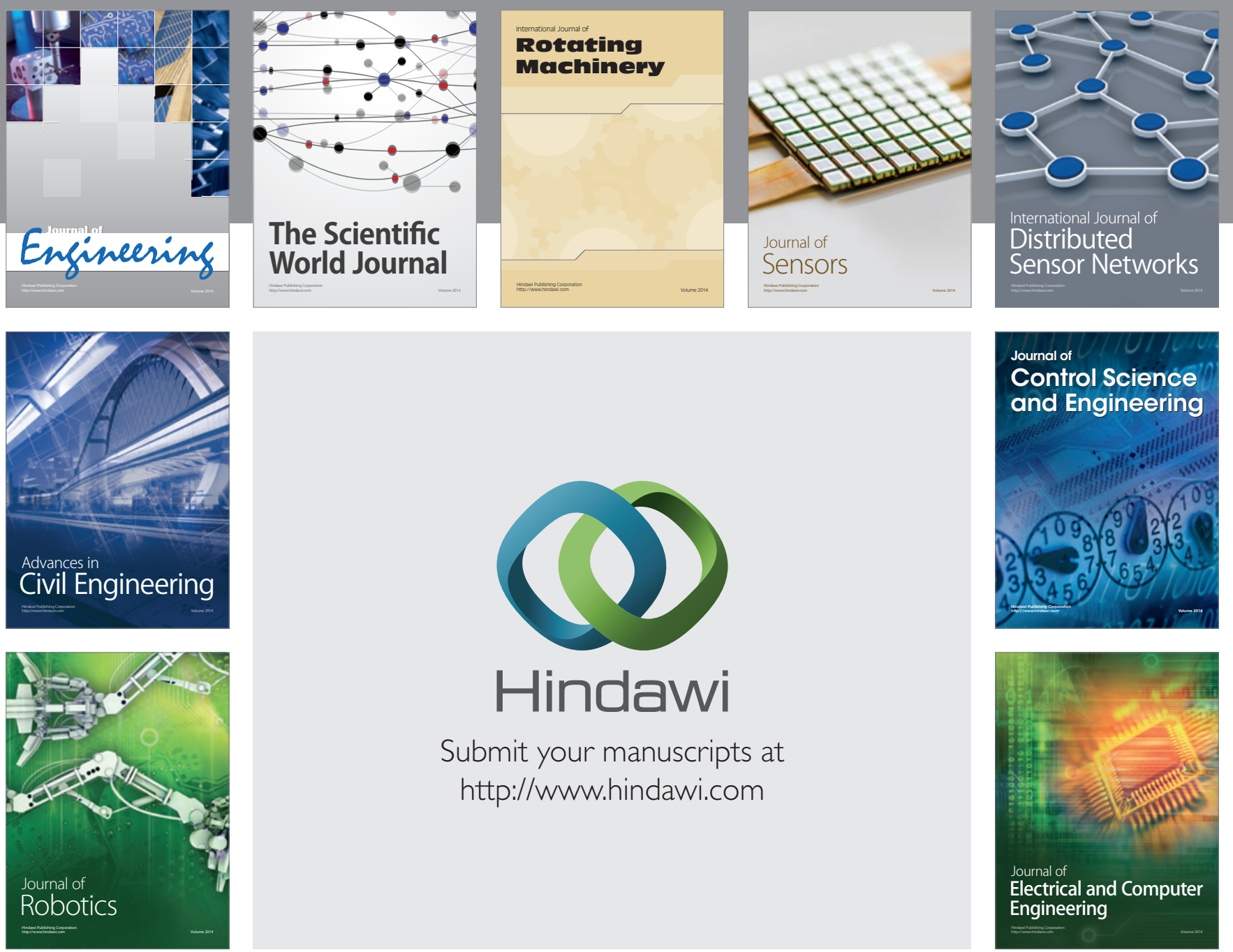

Submit your manuscripts at

http://www.hindawi.com
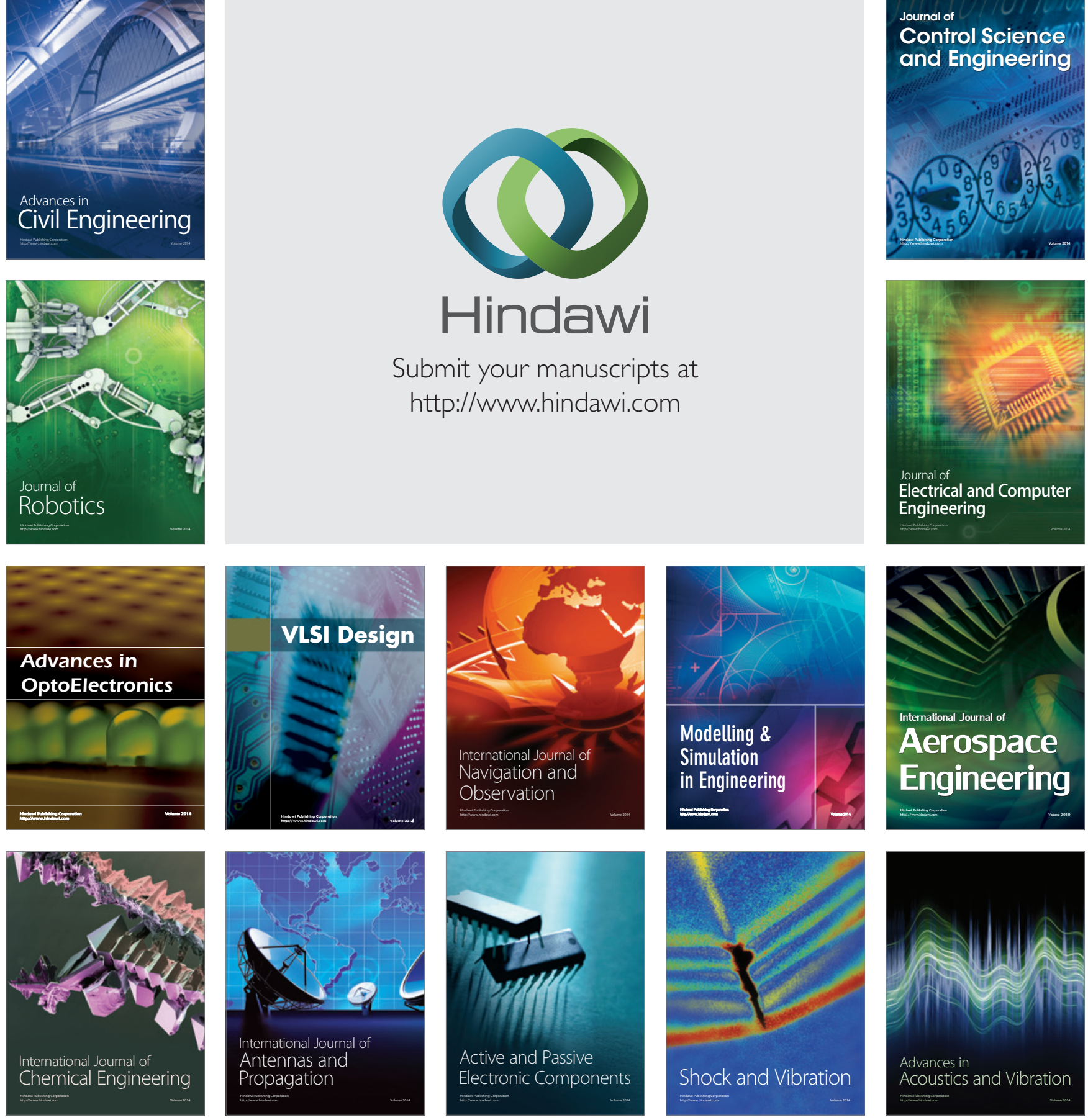